thelzellen des proximalen Tubulus. Die in diesen Zellen enthaltenen Enzyme gelangen in den Harn. Normalerweise stammt die LAP-Aktivität des Harnes aus dem Prozeß der Zellmauserung an den Tubuli; nach Auslösung von Schockreaktionen wird nun das gleiche Enzym hochgradig vermehrt ausgeschieden. Zweifellos ist dieser Mechanismus am Zustandekommen der erhöhten proteolytischen Aktivität des Harnes nach Auslösung allergischer Reaktionen (15-19) mitbeteiligt. Darüber hinaus kann auch durch das bei allergischen Reaktionen aktivierte Plasmin (18) eine Aktivierung von Nierenpeptidasen erfolgen (20).
In den vorliegenden Versuchen ergab sich eine lineare Korrelation zwischen der Abspaltung von $\beta$-Naphthylamin und p-Nitroanilin durch die LAP des Harnes. Diese lineare Korrelation fand sich nicht nur unter normalen Bedingungen, sondern sie war auch bei pathologisch gesteigerter Ausscheidung von renaler LAP nachweisbar. L-Leucyl-p-Nitroanilid ist also zur Aktivitätsbestimmung der renalen LAP ebensogut geeignet wie L-Leucyl- $\beta$-Naphthylamin. Es gilt aber auch hier die in der Einleitung gemachte Einschränkung, daß keineswegs eine ausschließliche Bestimmung der echten Leucinaminopeptidase durchgeführt wird.

\title{
Literatur
}

1. Spackman, D. H., E. L. Smith und D. M. Brown, J. biol. Chemistry 212, 255 (1955). - 2. HaNson, H., D. Glaesser und H. KirschKe, Hoppe-Seylers' Z. physiol. Chem. 340, 107 (1965). 3. NAGEL, W., F. Willig und F. H. Schmidr, Klin. Wschr. 42, 447 (1964). - 4. Green, M. N., K.-C. Tsou, R. Bressler und A. M. Seligman, Arch. Biochemistry 57, 458 (1955). - 5. Steigleder, G.-K., Y. Kamei und R. Kudicke, Arch. klin. exp. Derm. 217, 417 (1963). - 6. O'Conell, W. und R. J. WinzleR, Cancer Res. 23, 78 (1963). - 7. Tuppy, H., U. Wiesbauer und E. WintersBerger, Hoppe-Seyler's Z. physiol. Chem. 329, 278 (1962). 8. BergmanN, H. und F. Scheler, Klin. Wschr. 42, 275 (1964). 9. Klaus, D., Ärztl. Forsch., Wörishofen 15, 548 (1961). - 10. BergmanN, H. und F. Truss, Med. Welt, 1964, 1760. - 11.
RAAB, W., Allergie und Asthma, im Druck. - 12. RAAB, W.: Renal enzyme excretion following anaphylactic shock Nature (London) im Druck. - 13. RAAB, W., Hautarzt 14, 241 (1963). 14. RAAB, W. und E. KAISER, Experientia, Basel, 21, 720 (1965). 15. Damganrd, E. und G. Ungar, Amer. J. Physiol. 171, 717 (1952). - 16. UNGAR, G., T. YAMURA, J. B. Isola und S. Kobrin, J. Exper. Med. 113, 359 (1961). - 17. KAISER, E., W. RAAB und W. LEPIER, Arch. klin. exp. Derm. 220, 321 (1964). - 18. RAAB, W. und E. KAISER, Klin. Wschr. 43, 345 (1965). - 19. RAAB, W. und E. Karser, Allergie und Asthma, 11, 37 (1965). - 20. INNERfield, I., R. Harvey, F. Luongo und E. Blincoe, Proc. Soc. exp. Biol. Med. 116, 573 (1964).

Dr. Wolfgang Raab

Universitäts-Institut für Med. Chemie, 1090 Wien (Osterreich), Währingerstraße 10

\section{Elektrophorese in horizontalem Polyacrylamidgel}

\section{Mitteilung: Einfluß verscbiedener Elektrophoresebedingungen auf die Auflösung von Humanserum}

\author{
Von O. ZwisLer und H. BIEL \\ Aus der Bebringwerke $\stackrel{A}{ } \bar{G}$, Marburg/Labn \\ (Eingegangen am 14. Juli 1965)
}

\begin{abstract}
Es wird eine mit einfachen Mitteln durchführbare Methode der Elektrophorese in horizontal gelagertem, nicht gekühltem Polyacrylamid-Gel beschrieben. Der Einfluß von verschiedenen Puffern, der Gelkonzentration und des Quervernetzungsgrades, der Laufstrecke und der Proteinkonzentration auf die elektrophoretische Trennung von Humanserum wurde untersucht. Humanserum wird in mindestens 20 unterscheidbare Komponenten zerlegt.

A method employing simple materials for electrophoresis in horizontally layered, non-cooled polyacrylamide gel is described. The influence of different buffers, gel concentration, degree of cross linkage, running distance and protein concentration on the electrophoretic separation of human serum were studied. Human serum was separated into at least 20 different components.
\end{abstract}

Seit der Einführung von Polyacrylamidgel („PAA“-Gel) als Träger für Elektrophoresen durch RAYMOND und WeINTraub (1) hat sich dieses synthetische Gel als vielseitig verwendbar erwiesen (2). Die Trennung eines Eiweißgemisches in Polyacrylamidgel erfolgt wie in Stärke-

\footnotetext{
Abkürzungen: PAA-Gel = Polyacrylamid-Gel DMAPN $=$ Dimethylaminopropionitril AP $=$ Ammoniumpersulfat AC $=$ Acrylamid MBAC $=\mathrm{N}, \mathrm{N}^{\prime}$-Methylenbisacrylamid
}

gel nicht nur nach verschiedener Ladung, sondern auch nach dem Molekulargewicht, bzw. der Molekülkonfiguration der Komponenten, doch ist es im Gegensatz $z \mathfrak{u}$ Stärkegel einfacher und stets reproduzierbar darstellbar und gegen physikalische und chemische Einflüsse widerstandsfähiger.

Bei der Elektrophorese in PAA-Gel ist es üblich, in vertikal gelagerten Gelen zu arbeiten $(3,4,5)$. Eine Verzerrung der Zonen durch Erwärmung oder durch Austrocknung des Gels während des Elektrophoreselaufes 
wird in der handelsüblichen Spezialapparatur vermieden (6).

Wir untersuchten nur die Elektrophorese in horizontal angeordneten PAA-Gelen, wobei wegen der geringen angelegten Spannung eine Kühlung entfällt und somit die Elektrophorese in PAA-Gel mit einfachen Mitteln durchführbar wird (vgl. 8).

\section{Methodik}

Apparatur

Als Bodenplatten wurden $32-36 \mathrm{~cm}$ große Platten aus normalem Tafelglas verwendet, die mit einem $1 \mathrm{~cm}$ breiten Glasrand von $3 \mathrm{~mm}$ Höhe umklebt sind, sowie einer Deckplatte passender Größe. Als Puffertrog kann jede beliebige“Ausführung geeigneter Breite Verwendung finden.

Beschickung des PAA-Gels

$\mathrm{Zu} 300 \mathrm{ml}$ einer 6,6 proz. Cyanogum-Lösung - Cyanogum 41 American Cyanamid Comp. - in Puffer werden je $30 \mathrm{ml}$ einer 6,6 proz. DMAPN-Lösung und $30 \mathrm{~m} /$ einer 0,6 proz. AP-Lösung unter Rühren zugegeben und in die waagerecht gelagerte Bodenplatte gegossen. Durch Auflegen der Deckplatte wird der Flüsssigkeitsüberschuß luftblasenfrei entfernt.

Um eine Auftragsstelle zu erhalten, die ein strähnenfreies Abwandern der Proteine vom Papier in das Gel gestattet, wurde vorher auf die Unterseite der Deckplatte etwa $5-7 \mathrm{~cm}$ vom Kathodenende entfernt ein 1,5 mm hoher, bis zu $20 \mathrm{~mm}$ tiefer Agarstreifen (1,0\% Reinagar Behringwerke in Gelpuffer) gelegt, der quer über die gesamte Deckplattenbreite reicht.

Nach etwa 1 Stunde Lagern - die Polymerisation ist bereits nach 10 Min. beendet - wird die Kammer geöffnet und der Agarstreifen aus dem $\mathrm{Gel}$ herausgenommen. Filtrierpapierstreifen $(0,6-1,8 \mathrm{~cm}$ Tiefe, 3-4 cm Breite, Schleicher \& Schüll Nr. 2315) werden mit der zu untersuchenden Lösung befeuchtet und auf die leicht klebrige Auftragsstelle gelegt (Abb. 1).

Bei konstanter aufgetragener Proteinmenge beeinflußt die Tiefe des Papierstreifens in den angegebenen Grenzen die Trennschärfe nicht.

Elektrophorese

Zum Schutz vor Austrocknung wird über die Auftragsrinne ein etwa $3 \mathrm{~cm}$ breiter Celluloidstreifen gelegt und, nachdem die Platte durch Filtrierpapierstreifen mit den gefüllten Puffertrögen verbunden ist, das gesamte Gel mit einer dünnen PVC-Folie bedeckt. Nach Auflegen einer $2 \mathrm{~mm}$ dicken Gummiplatte liegt die Folie
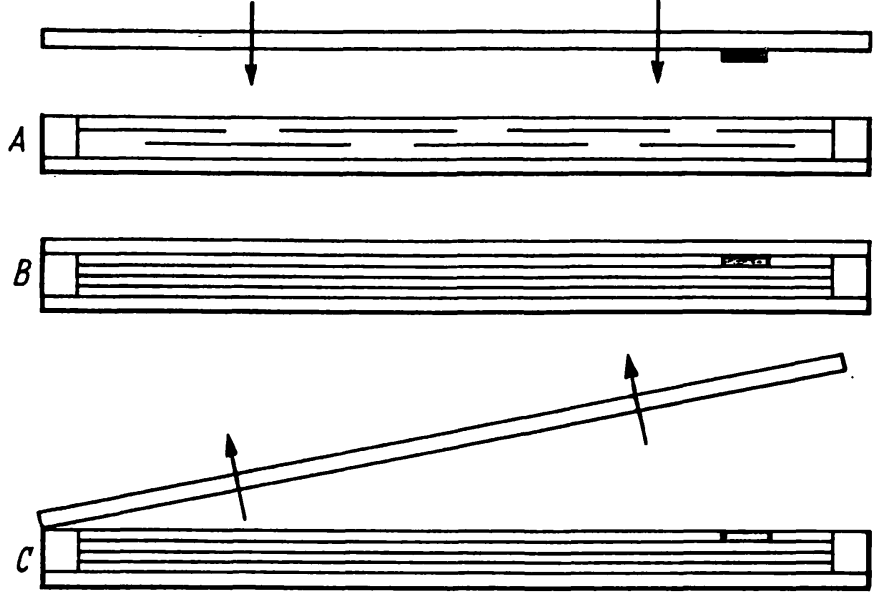

Abb. 1

Einbringen des Agarstreifens

A: Auflegen der Deckplatte mit Agarstreifen

B: Einpolymerisieren des Agars

C: Agarstreifen einpolymerisiert (Auftragsstelle)

glatt auf dem Gel, so daß ein Bestreichen des Gels mit Silikonlack nicht mehr notwendig ist (7).

Danach werden 3,3 V/cm angelegt; nach 15 bis 16 Stunden ist die Boratfront, kenntlich an einer leichten Verfärbung quer zur Wanderungstichtung (Verunreinigung des Cyanogums?) 20 bis $25 \mathrm{~cm}$ weit eingewandert, so daß der Lauf abgebrochen werden kann.

Färbung des PAA-Gels

Nach Abnehmen der Deckfolien wird die Gelschicht von der Trägerplatte abgelöst und $2 \frac{1}{2}$ Stunden unter leichtem Hin- und Herbewegen mit Amidoschwarz in üblicher Weise gefärbt. Die Entfärbung erfolgt mit Methanol/Wasser/Essigsäure (5:5:1) über Nacht, das durch ein Aktivkohlefilter zirkuliert. - Zur Dokumentation werden die entfärbten Gelplatten in einen von unten beleuchteten Milchglastrog gelegt und im Durchlicht fotografiert.

\section{Ergebnisse}

\section{Wahl des Puffers}

Nach Prüfung verschiedener Puffersysteme auf ihre Brauchbarkeit wurden nach Vorversuchen fünf Puffer in engere Wahl gezogen (Tab. 1). Bei annähernd gleichem

Tab. 1

Ansatz der Gelpufferlösungen

\begin{tabular}{llcccc}
\hline Pufferart & Konzentration & IV & III & II & I \\
& & $(150 \%)$ & $(100 \%)$ & $(50 \%)$ & $(10 \%)$ \\
\hline
\end{tabular}

Veronalpuffer

Stammlösung: Diäthylbarbitursaures Natrium 0,08m + Diäthylbarbịtursäure 0,02 m Nach Cyanogumzusatz mit $\mathrm{H}_{2} \mathrm{O}$ auf $1 /$ aufgefüllt

$450,0 \mathrm{ml} \quad 300,0 \mathrm{ml}$

$150,0 \mathrm{ml}$

$30,0 \mathrm{ml}$

Trispuffer Stammlösung: $51,5 \mathrm{~m} l$ Tris $2,54 m+6,0 \mathrm{ml}$ Citronensäure $2,54 m$ Nach Cyanogumzusatz
mit $\mathrm{H}_{2} \mathrm{O}$ auf $1 /$ aufgefüllt

$39,0 \mathrm{ml} \quad 26,0 \mathrm{ml}$

$13,0 \mathrm{ml}$

$2,6 \mathrm{ml}$

Tris-Boratpuffer*)

a) Trispuffer: $51,5 \mathrm{ml}$ Tris 2,54 m+6,0 $\mathrm{ml}$ Citronensäure $2,54 \mathrm{~m}$

b) Boratpuffer: $13,17 \mathrm{ml} \mathrm{H}_{3} \mathrm{BO}_{3} 1,094 \mathrm{~m}+12,4 \mathrm{ml} \mathrm{NaOH} 1,094 m$

Stammlösung: $12,2 \mathrm{~m} /$ Trispuffer a) $+4,95 \mathrm{ml}$ Boratpuffer $\mathrm{b}$ )

Nach Cyanogumzusatz

mit $\mathrm{H}_{2} \mathrm{O}$ auf 1 l aufgefüllt $\quad 51,4 \mathrm{ml} \quad 34,3 \mathrm{ml}$

$17,6 \mathrm{ml}$

$3,42 \mathrm{ml}$

Boratpuffer

Stammlösung: $31,17 \mathrm{~m} l \mathrm{H}_{3} \mathrm{BO}_{3} 1,094 m+12,4 \mathrm{~m} l \mathrm{NaOH} 1,094 m$

Nach Cyanogumzusatz

mit $\mathrm{H}_{2} \mathrm{O}$ auf $1 /$ aufgefüllt

$120,0 \mathrm{ml}$

$80,0 \mathrm{ml}$

$40,0 \mathrm{ml}$

$.8,0 \mathrm{ml}$

*) $\mathrm{V}=200 \%(68,6 \mathrm{~m} / \mathrm{T}$ Tris-Borat-Stammlösung auf 1 l). 
$\mathrm{pH}(8,6-8,8)$ wurden sie auf gleiche Leitfähigkeit eingestellt (Konz. III [100\%] in Tab. 1). Sie dienten zum Lösen des Cyanogums (Gel-Puffer).

Als Elektrodenpuffer verwendeten wir für die Versuche folgende in Tabelle 2 aufgeführten Lösungen:

Tab. 2

Elektrodenpufferlösungen

\begin{tabular}{|c|c|}
\hline Gelpuffer & Elektrodenpuffer \\
\hline Veronalpuffer & $\begin{array}{l}\text { Veronalpuffer: Diäthylbarbitursaures Natri- } \\
\text { um } 0,08 m+\text { Diäthylbarbitursäure } 0,2 m\end{array}$ \\
\hline Tris-Puffer & $\begin{array}{l}\text { Boratpuffer: } 3,71 l \mathrm{H}_{3} \mathrm{BO}_{3} 1,094 m 1,24 l \\
\mathrm{NaOH} 1,094 m ; 1+2 \text { mit } \mathrm{H}_{2} \mathrm{O} \text { verdünnt } \\
\text { Trispuffer: } 448 \mathrm{~m} l \text { Tris } 2,54 \mathrm{~m}+52 \mathrm{~m} l \\
\text { Citronensäure } 2,54 m \text { auf } 2,0 l \mathrm{H}_{2} \mathrm{O}\end{array}$ \\
\hline Tris-Borat-Puffer & $\begin{array}{l}\text { Boratpuffer: } 3,71 l \mathrm{H}_{3} \mathrm{BO}_{3}+1,24 l \mathrm{NaOH} \\
1,94 m ; 1+2 \text { mit } \mathrm{H}_{2} \mathrm{O} \text { verdünnt }\end{array}$ \\
\hline Borat-Puffer & $\begin{array}{l}\text { Boratpuffer: } 3,71 l \mathrm{H}_{3} \mathrm{BO}_{3} 1,094 m+1,24 l \\
\mathrm{NaOH} 1,094 m ; 1+2 \text { mit } \mathrm{H}_{2} \mathrm{O} \text { verdünnt }\end{array}$ \\
\hline
\end{tabular}

Bei Verwendung der in Tabelle 1 angegebenen Puffer und der in Tabelle 2 angeführten Elektrodenpuffer erhielten wir sehr unterschiedliche Auftrennungen. Das für jeden Puffer optimale Ergebnis zeigt Abbildung 2.

Im Falle des Tris-Boratpuffers, der das günstigste Trennergebnis zeigt, wird auch bei Variierung der Pufferkonzentrationen die Auffächerung nicht mehr verbessert (Abb. 3). Unter Beibehaltung der kürzlich vorgetragenen Benennung einzelner Abschnitte des Elektrophoresefeldes nach dort befindlichen repräsentativen Proteinen (7) ist die Auftrennung bei Verwendung dieses Puffers im Postalbumin-, Hämopexin- und Posttransferrinbereich gleichermaßen befriedigend. Dieser Puffer wurde für sämtliche weiteren Versuche verwendet.

Zur Ermittlung der optimalen Gelkonzentrationen wurden Gele verschiedener Dichte hergestellt. Diese Er-

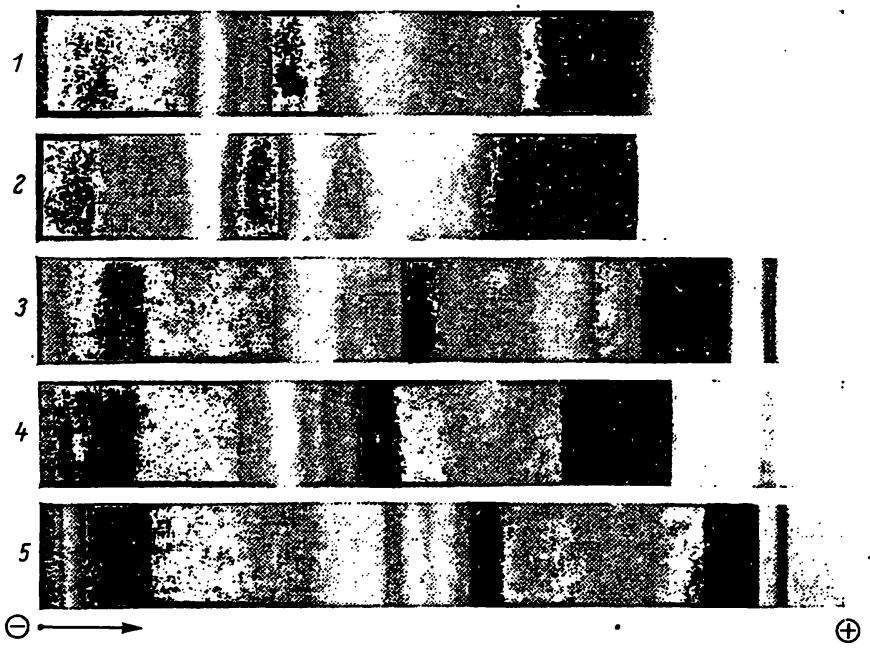

Abb. 2

$\oplus$

Einfluß verschiedener Puffer auf die Trennung von Serumproteinen in PAA-Gel
$1=$ Veronal/Veronal-Puffer
$2=$ Tris/Tris-Puffer
3 = Tris-Borat/Borat-Puffer (Standard)
$4=$ Borat/Borat-Puffer
$5=$ Tris/Borat-Puffer

gebnisse sind aus Abbildung $4 \mathrm{zu}$ ersehen. Eine Erhöhung der Konzentration über 5,5\% hinaus ergibt bei gleichzeitiger Verminderung der Wanderungsgeschwindigkeiten der Proteine und der damit verbundenen schlechteren Auflösung lediglich eine verbesserte Trennung Albumin/saures $\alpha_{1}$-Glykoprotein.

$\mathrm{Da}$ nach Verwendung von Cyanogum 41 bereits ein festes Verhältnis von Polymeren zu Copolymeren im PAA-Gel vorgegeben ist, wurde durch Mischen beider Monomeren (Acrylamid und N,N'-Methylenbisacrylamid) Polyacrylamid mit verschieden starkem Vernetzungsgrad bei gleicher Endkonzentration von 5,5\% hergestellt. Die Trennungsergebnisse, die mit diesen Gelen erhalten wurden, zeigt Abbildung 5. Im Posttransferrinbereich kann durch verșchieden starke Quervernetzung die Trennung leicht verbessert werden.

Optimale Trennung findet nur unter Ausnutzung der gesamten Weglänge statt. Wie in Abbildung $6 \mathrm{zu}$ ersehen, ist es zweckmäßig, anodisch wanderndes Material möglichst in Kathodennähe aufzutragen, während Abbildung 7 die Ausbildung scharfer Banden und die Erhöhung der

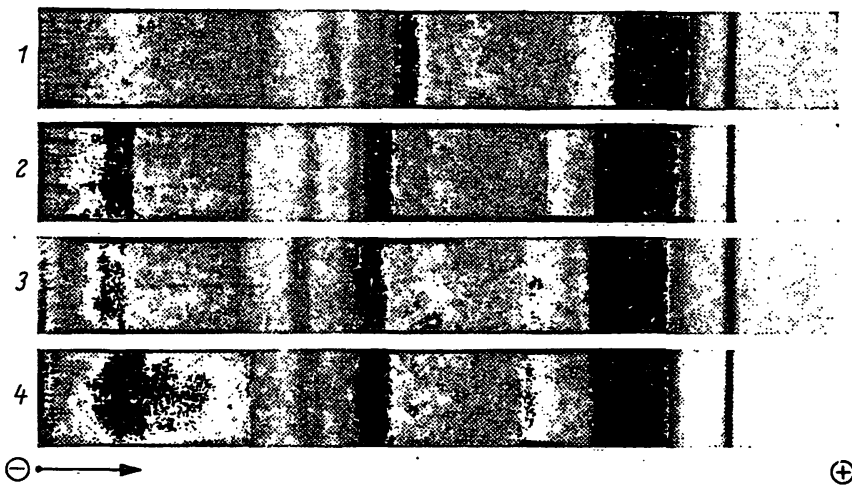

Abb. 3

Einfluß der Konzentration des Gelpuffers (Tris/Borat-Puffer) auf die Trennung von Serumproteinen in PAA-Gel

1: II $(50 \%)$ in Tab. $1 \quad 3:$ IV (150\%) in Tab. 1

2: III $(100 \%)$ in Tab. 14 V $(200 \%)$ in Tab. 1

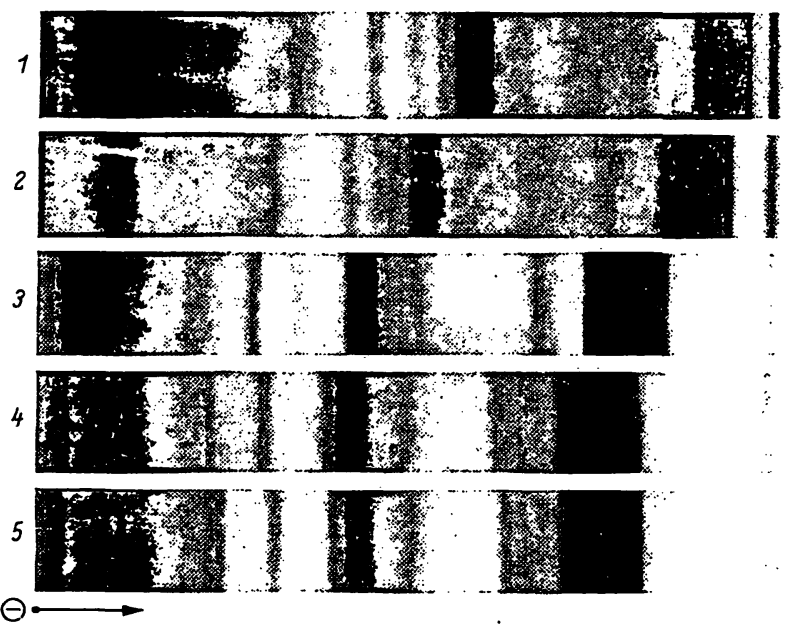

$\oplus$

Abb. 4

Einfluß der Gelkonzentration auf die Trennung von Serumproteinen in PAA-Gel

$$
\begin{array}{cr}
1=4,5 \% \text { Cyanogum } & 3=6,5 \% \text { Cyanogum } \\
2=5,5 \% \text { Cyanogum } & \begin{array}{r}
3=7,5 \% \text { Cyanogum } \\
5=8,5 \%
\end{array} \\
& \text { Cyanogum }
\end{array}
$$



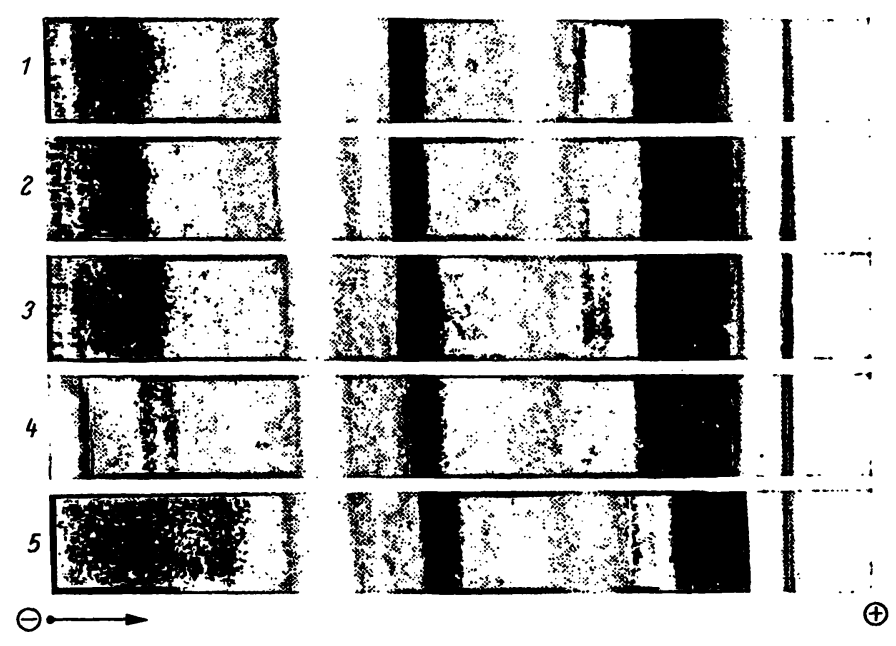

Abb. 5

Einfluß der Quervernetzung in 5,5 proz. PAA-Gel auf die Trennung von Serumproteinen

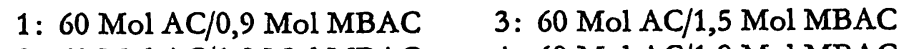

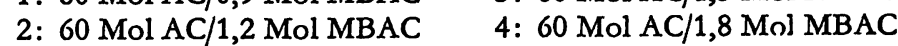
5: Cyanogum 41

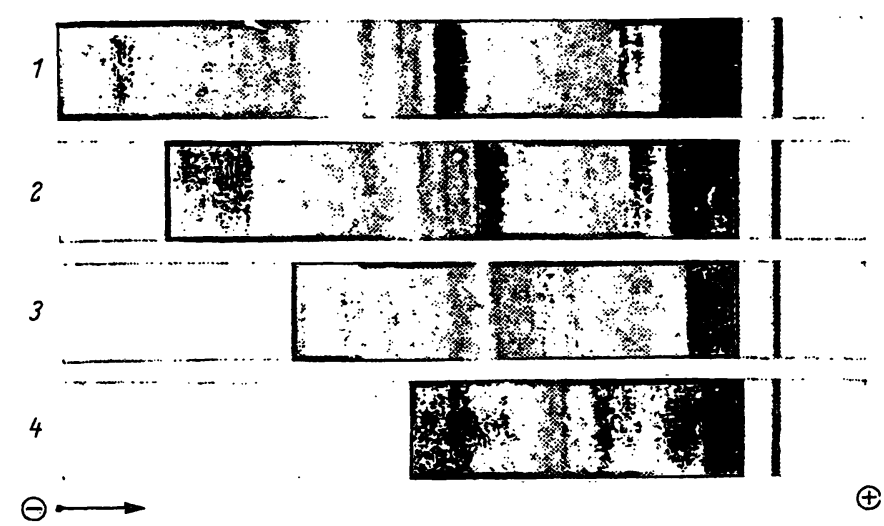

Abb. 6

Einfluß der Laufstrecke auf die Trennung von Serumproteinen Entfernung der Auftragstelle vom Kathodenende $\begin{array}{llll}1: 4 \mathrm{~cm} & 2: 8 \mathrm{~cm} & 3: 12 \mathrm{~cm} & 4: 16 \mathrm{~cm}\end{array}$

Trennschärfe in Abhängigkeit von dem Einwandern einer diskontinuierlichen Pufferfront zeigt. In $\mathrm{Ab}$ bildung 8 ist der Einfluß der Proteinkonzentration auf die Trennbarkeit und auf die Nachweisbarkeit der Einzelkomponenten dargestellt.

\section{Diskussion}

Die Elektrophorese in PAA-Gel wird in üblicher Weise in vertikal angeordneten Gelen durchgeführt $(3,6)$, doch sind auch horizontal gelagerte Gele einfach zu bereiten (8).

In der von uns gewählten horizontalen Versuchsanordnung wurde der Einfluß von 5 verschiedenen Puffersystemen in verschiedenen Konzentrationen auf die Trennung von Serumproteinen untersucht.

Wie bereits für die Stärkegelelektrophorese gezeigt (9), ist auch in Polyacrylamid die Verwendung eines diskontinuierlichen Tris-Boratsystems empfehlenswert, da es sich in der Trennschärfe anderen von uns untersuchten Puffersystemen überlegen zeigt (vgl. Abb. 1).

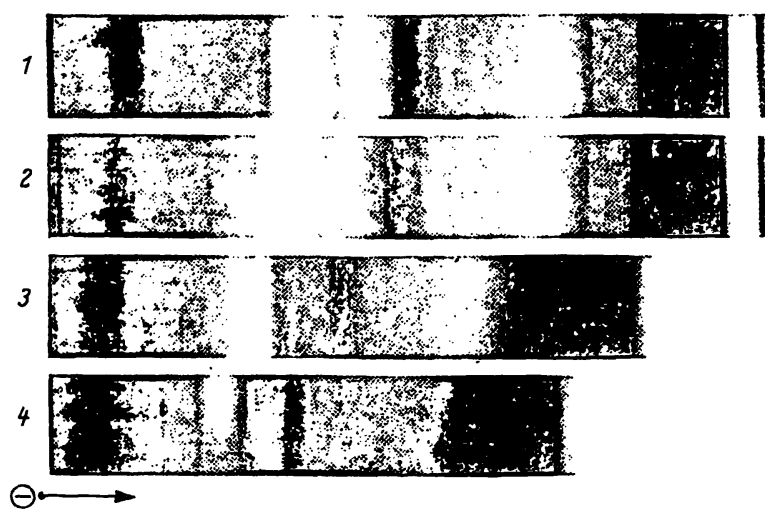

$\oplus$

Abb. 7

Einfluß der einwandernden Boratfront auf die Trennung von Serumproteinen in PAA-Gel

Auftragung der Substanz vor, während und nach der Uberwanderung der Auftragstelle

1: $3 \mathrm{~cm}$ vor Uberwanderung $\quad 3: 3 \mathrm{~cm}$ nach Uberwanderung 2: $0 \mathrm{~cm}$ vor Ưberwanderung $\quad 4: 6 \mathrm{~cm}$ nach Überwanderung

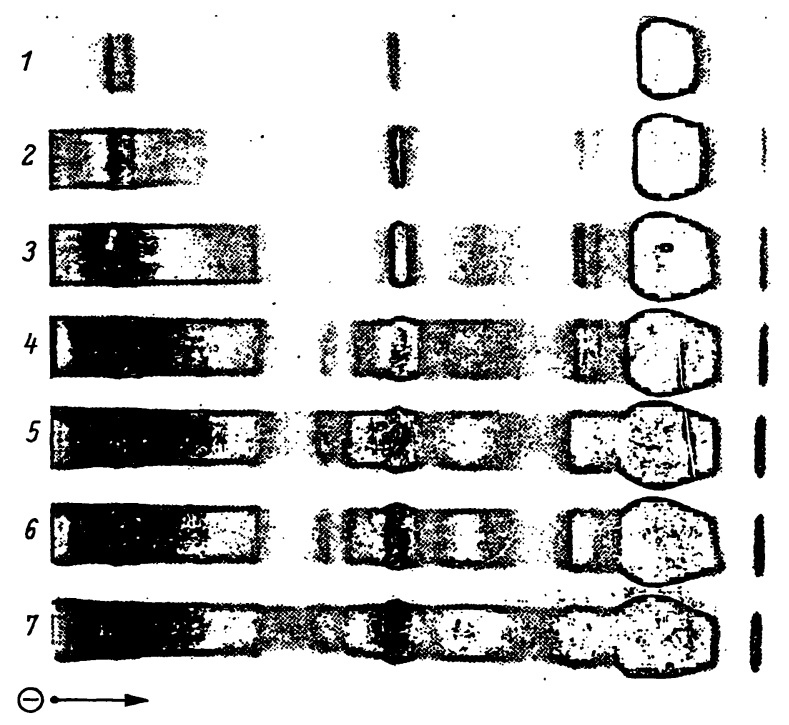

Abb. 8

Einfluß der Konzentration der Serumproteine auf Trennung und Nachweisbarkeit der Einzelkomponenten

$$
\begin{array}{ll}
1=0,5 \mathrm{mg} \text { Protein } & 4=1,9 \mathrm{mg} \text { Protein } \\
2=1,0 \mathrm{mg} \text { Protein } & 5=2,4 \mathrm{mg} \text { Protein } \\
3=1,4 \mathrm{mg} \text { Protein } & 6=2,9 \mathrm{mg} \text { Protein }
\end{array}
$$

$7=3,5 \mathrm{mg}$ Protein

Durch Erhöhen der Gelkonzentration wandert das saure $\alpha_{1}$-Glykoprotein aus der Albuminbande aus (Abb. 3, vgl. (7)). In keinem Fall jedoch gelingt die Trennung Albumin- $\alpha_{1}$-Antitrypsin.

Aus den Abbildungen 1 und 2 ist zu ersehen, daß die beste Trennung der Komponenten in Postalbumin- und Hämopexinbereich erhalten wird, während im Posttransferrinbereich die Trennung unvollkommen ist. Bei Trennungen in diesem Bereich zeigt die Elektrophorese in Stärkegel bessere Ergebnisse.

Obwohl die Quervernetzung kaum die Porengröße des PAA-Gels beeinflußt (10), zeigt PAA-Gel mit unterschiedlich starkem Quervernetzungsgrad in verschiede- 
nen Bereichen leichte Unterschiede im Auflösungsvermögen. Sofern jedoch nicht eine besondere Problemstellung vorliegt, ist das Trennvermögen von käuflichem Cyanogum 41 ausreichend. Von Bedeutung für die
Trennung ist dagegen die Ausnutzung der vollen Laufstrecke sowie das Einwandern der konzentrierten Boratfront des Elektrodenpuffers, die das Ausbilden scharfer schmaler Zonen bedingt.

\title{
Literatur
}

1. Raymond, S. und L. Weintraub, Science (Washington) 130, 711 (1959). - 2. E-C Apparatus. Corp., Techn. Bulletin Nr. 125-B, Literaturübersicht bis 1963, s. auch Brel, H., N. HeimbUrger., D. Kraft, Th. KRANZ und R. Schmidtberger, Behringwerk-Mitt, 43, 1 (1964). - 3. RAYMond, S. und Y. WANG, Analyt. Biochem. (New York) 1, 391 (1960). - 4. RaYMOND, S. und M. NaKAMICHr, Analyt. Biochem. (New York) 3, 23 (1962). - 5. Otr, H. und S. Marinis in Protides of the Biol. Fluids, S. 408. 11th Coll. Brügge
1963, hrsg. von H. Peeters, Elsevier Publ., Amsterdam (1964). 6. Electrophoresis apparatus; E-C Apparatus Corp., 22250 40th Street, University City, Philadelphia 4, PA, USA. - 7. ZwrsLER, O. und H. Brel in: Protides of the Biol. Fluids, S. 433. 12th Coll. Brügge 1964, hrsg. von H. Peeters, Elsevier Publ. Amsterdam (1965). - 8. Mourray, K., Biochim. Biophys. Acta 59, 211 (1962). - 9. Pourrk, M. D., Nature (London) 180, 1477 (1957). - 10. Whrte, M. L., J. phys. chem. 64, 1563 (1960).

Dr. O. Zwisler

Behringwerke AG

335 Marburg/Lahn

\section{Zur Frage der Tagesschwankungen des Serumcholesterins}

\author{
Von CH. StöckiI \\ Aus dem Forscbungslaboratorium der. Neurologischen Universitätsklinik Basel (Direktor: Prof. H. E. Kaeser)
}

(Eingegangen am 9. Juli 1965)

\begin{abstract}
Die Cholesterinbestimmungen wurden mit einer eigenen Ultramikromodifikation der spektrokolorimetrischen Methode nach Huang vorgenommen. Um die Kurve der Tagesschwankungen des Serumcholesterins zu erhalten, wurden pro Versuchsperson, in der Zeit von 8.00-19.00 in stündlichem Abstand 12 Bestimmungen vorgenommen. Das Probandengut bestand aus 15 gesunden Personen, 6 Frauen und 9 Männer zw. 18-56, mit einem Durchschnittsalter von 37 Jahren.

Bei diesen Versuchspersonen konnten rhythmische Tagesschwankungen von gesetzmäßigem Charakter nachgewiesen werden. Darunter gibt es Individuen mit geringen und solche mit beträchtlichen Tagesschwankungen. Nimmt man als Ausmaß der Cholesterin-Tagesschwankung den größten minus den kleinsten Tageswert, so zeigt die labilste Versuchsperson eine solche von $66 \mathrm{mg} / 100 \mathrm{ml}$, die stabilste jedoch nur eine von $6 \mathrm{mg} / 100 \mathrm{~m} /$. Die 3 verglichenen Kurven zeigen übereinstimmend einen morgendlichen Cholesterinanstieg zu einem Hochpunkt, der in die Mittagszeit fällt, und einen anschließenden nachmittäglichen Abfall. Für die Klinik ergibt sich, daß eine optimale Erfassung des Cholesterinspiegels, seiner Schwankungsrichtung und -breite am besten mit einer Drei-Zeit-Messung um 8, 12 und 15 Uhr gewährleistet ist.
\end{abstract}

An ultramicromodification was made to the spectrophotometric method of HuaNG for cholesterol determination. In order to obtain a curve of the daily variation of serum cholesterol, 12 hourly determinations were made on each subject between 8 a. m. and 7 p. m.; 15 healthy persons, 6 women and 9 men, average age 37 (range 18-56) were studied.

In these experiments regular, rythmic, daily variations were found. Some persons showed small and others large daily variations. If the difference between the highest and the lowest daily level is taken as a measure of the daily variation of cholesterol, the most labile subject showed a difference of $66 \mathrm{mg} / \mathrm{ml}$, while the most stable one showed a value of $6 \mathrm{mg} / 100 \mathrm{~m} /$. Each of three comparable curves shows in the morning hours an increase in cholesterol to a maximum during midday which decreases again in the afternoon. For clinical purposes, the optimal determination of the cholesterol level and the direction and extent of its variation is best achieved by three determinations at 8 a. $\mathrm{m}$., noon, and 3 p. m.

In Anbetracht des großen Interesses, welches das Serumcholesterin nicht nur von Seiten der Arterioskleroseforschung, sondern auch in der neurologischen und psychiatrischen Medizin erfahren hat $(1-8,20)$, ist eine neuerliche Abklärung der Frage, ob es sich bei diesem Stoff um einen statischen Parameter handelt, oder ob er gewissen kurzfristigen Tagesschwankungen unterworfen ist, angezeigt.

Aus internistischen Untersuchungen bis etwa 1926 schien hervorzugehen, da $\beta$ die Konzentration des Serumcholesterins beim Gesunden praktisch konstant sei, so- fern nicht durch alimentäre Belastung eine in wenigen Stunden abklingende Erhöhung eintrete. Serienuntersuchungen durch GeORGI von 1926-33 mit 2161 Cholesterin-Bestimmungen bei 456 depressiven Patienten und 21 Gesunden (3) führten jedoch u. a. zur Feststellung, daß physisch und psychisch gesunde Männer keine nennenswerten Schwankungen während Wochen und Monaten aufwiesen, wenn die Blutentnahme zur gleichen Tagesstunde vorgenommen wurde (gesunde Frauen zeigten größere Schwankungen im Praemenstrum). Im Gegensatz zu psychisch Gesunden wurden bei 